\title{
An Optimized Unbiased GM $(1,1)$ Power Model for Forecasting MRO Spare Parts Inventory
}

\author{
Chen Bing ${ }^{1}$, Sun Shouqun ${ }^{1} \&$ Liu Gang ${ }^{2}$ \\ ${ }^{1}$ School of Mechanical Engineering, University of Shanghai for Science and Technology, Shanghai, China \\ ${ }^{2}$ Shanghai Baosteel Equipment Maintenance CO., Ltd., Shanghai, China \\ Correspondence: Chen Bing, School of Mechanical Engineering, University of Shanghai for Science and \\ Technology, Room 501, Building 9, NO. 334, Jungong Road, Yangpu District, Shanghai 200093, China. Tel: \\ 86-139-1762-4449. E-mail: chenbing25@gmail.com
}

Received: February 21, $2012 \quad$ Accepted: April 28, $2012 \quad$ Online Published: May 12, 2012

doi:10.5539/mas.v6n6p12 URL: http://dx.doi.org/10.5539/mas.v6n6p12

This work is supported by the National Nature Science Foundation NO. 50875174 and National 863 Plan NO. 2009AAllZ211

\begin{abstract}
With respect to the problem of complexity and uncertainty in the MRO (Maintenance, Repair and Overhaul) spare parts inventory, an optimized grey forecasting model is employed to forecast the demand of spare parts. The parameter $\gamma$ is optimized based on genetic algorithm (GA) method in the unbiased GM $(1,1)$ power model to minimize the ARPE (Average Relative Proportional Error) of accuracy. And the optimal model is used to forecast the prediction demand in a practical example. The experiment results indicate the forecasting accuracy can be accepted by using the optimized unbiased GM $(1,1)$ power model.
\end{abstract}

Keywords: MRO, grey prediction, unbiased GM $(1,1)$ Power Model, GA method, spare parts inventory

\section{Introduction}

In the lifecycle of continuous casting equipment, the comprehensive digital solutions and informationization integration technology are employed by MRO (Maintenance, Repair and Overhaul/ Operation) (Garg A., 2006) supporting system for equipment manufacturing enterprise, equipment user enterprises, and equipment service enterprises. MRO system plays an important role in keeping the continuous casting equipments operating efficiently and stably. However the demand and order period of MRO spare parts are uncertain. Besides uncertainty, the funds and cost constraint also are serious facts that have an influence on the level of MRO service. Because of these facts, the inventory cost and purchase cost of MRO spare parts are kept on a high level (Yang Kelei, 2009). Demand forecasting for MRO spare parts inventory not only improving the inventory service standard and decreasing the direct cost of inventory (John E. Boylan, 2010), but also helping the steel enterprise to reduce the outage time, raise the production efficiency, and enhance the competitive of steel enterprise greatly (Wang Wenbin, 2011).

At present, there are many forecasting methods of demand for spare parts inventory include: regression analysis, time series prediction, exponential smoothing, Bayesian method (Kostas-Platon Aronis, 2004), neural network (Amin-Naseri, M. R., 2008), grey prediction etc. Grey prediction is a prediction method based on gray system theory without large samples. The uncertain system with small sample and poor information is regarded as grey prediction's research object. By using a short sequence of raw data to create dynamic forecasting model, a high precision of prediction could be got (Deng Julong, 2002). GM $(1,1)$ model is the basic model of grey prediction, it is widely used in industry (Li Derchiang, 2009), agriculture, education, military (Chiou Huakai, 2004), economic (Wang Yifan, 2002), and the other fields (Zhang Jiantong, 2009). GM (1, 1) power model is a kind of non-linear grey model, and is the expansion of traditional GM $(1,1)$ model and grey Verhulst model. The recursive method of unbiased grey prediction model was proposed by Shi Bin et al. The recursive prediction formulas for unbiased gray model were provided under different conditions, and the problem of optimization model was studied (Shi Bin, 2011). In order to eliminate the inherent deviation of the traditional GM $(1,1)$ power model, the unbiased GM $(1,1)$ power model was constructed and the problem of the optimization of the 
parameters in the model was studied by Wang Zhenxin et al. It was proved that the forecast accuracy could be improved significantly by using unbiased GM $(1,1)$ power mode (Wang Zhenxin, 2011).

This paper consider the problem of low forecast accuracy for MRO Spare Parts Inventory of continuous casting equipment in steel enterprise. The parameter of unbiased GM $(1,1)$ power model is optimized by using the genetic algorithm, and a grey model is constructed according to the historical demand data of MRO spare parts inventory. The demand forecast accuracy is improved significantly.

\section{Optimization of Unbiased GM $(1,1)$ Power Model}

\subsection{Unbiased GM $(1,1)$ Power Model}

For rigorous statements, the following definitions and Theorem of unbiased GM $(1,1)$ power model are going to be represented according to Wang Zhengxin et al's research. The complete contents can be access from relevant literature (Wang Zhenxin, 2011).

Definition 1. Assume that $\hat{X}^{(0)}=\left(\hat{x}^{(0)}(1), \hat{x}^{(0)}(2), \ldots, \hat{x}^{(0)}(n)\right)$ is the sequence of simulation of the grey model for the original sequence $\hat{X}^{(0)}, \hat{X}^{(0)}=\left(\hat{\hat{x}}^{(0)}(1), \hat{\hat{x}}^{(0)}(2), \ldots, \hat{\hat{x}}^{(0)}(n)\right)$ is the original sequence of the grey model, $\hat{X}^{(0)}$ is the new sequence of simulation which is from the original sequence, for any $k \in\{1,2, \ldots, n\}$, let $\hat{\hat{x}}^{(0)}(k)=\hat{x}^{(0)}(k)$, then this grey model is called unbiased grey model.

Definition 2. Assume that $X^{(0)}$ is the original sequence, $X^{(1)}$ is the 1-AGO sequence of $X^{(0)}$, if there is a sequence $Y^{(l)}=\left(y^{(l)}(1), y^{(l)}(2), \ldots, y^{(l)}(n)\right)$ let

$$
y^{(l)}(k)=\left[x^{(l)}(k)\right]^{l-\gamma}, \gamma \neq 1, k=1,2, \ldots, n .
$$

then we call $Y^{(1)}$ is the $1-\gamma$ times power generate sequence of $X^{(1)}$.

Definition 3. Assume that $X^{(0)}$ is the original sequence, $X^{(1)}$ is the 1-AGO sequence of $X^{(0)}, \quad Y^{(1)}$ is the $1-\gamma$ times power generate sequence of $X^{(1)}$, then

$$
\left\{\begin{array}{l}
y^{(l)}(k)=\beta_{1} y^{(I)}(k-1)+\beta_{2} \\
y^{(l)}(k)=\left[x^{(I)}(k)\right]^{1-\gamma} \\
\gamma \neq 1, k=2,3, \ldots, n .
\end{array}\right.
$$

is called unbiased $\mathrm{GM}(1,1)$ power model.

If $\hat{\beta}=\left(\beta_{1}, \beta_{2}\right)^{T}$ act as the parameter vector of the unbiased $\mathrm{GM}(1,1)$ power model, then the least-squares estimation parameters of the unbiased grey model is

$$
\hat{\beta}=\left(B^{T} B\right)^{-1} B^{T} Y
$$

Hereinto, $Y=\left[\begin{array}{c}y^{(I)}(2) \\ y^{(I)}(3) \\ \vdots \\ y^{(I)}(n)\end{array}\right], B=\left[\begin{array}{cc}y^{(I)}(1) & 1 \\ y^{(I)}(2) & 1 \\ \vdots & \vdots \\ y^{(l)}(n-1) & 1\end{array}\right]$

Theorem 1. Assume $B, Y, \quad \hat{\beta}$ as mentioned above, $\hat{\beta}=\left(B^{T} B\right)^{-1} B^{T} Y$, take the initial value, $\hat{x}^{(l)}(1)=x^{(l)}(1)$ the solution of the unbiased GM $(1,1)$ power model will be:

$$
\hat{x}^{(l)}(k)=\left\{\begin{array}{l}
{\left[\beta_{1}^{k-1}\left(x^{(0)}(1)\right)^{1-\gamma}+\frac{1-\beta_{1}^{k-1}}{1-\beta_{1}} * \beta_{2}\right]^{\frac{1}{1-\gamma}}\left(\beta_{1} \neq 1\right)} \\
{\left[\left(x^{(0)}(1)\right)^{l-\gamma}+(k-1) \beta_{2}\right]^{\frac{1}{1-\gamma}}\left(\beta_{1}=1\right)}
\end{array}\right.
$$

And then make another regressive reduction, the final analog and predictive value will be calculated. 


$$
\hat{x}^{(0)}(k)=\hat{x}^{(1)}(k)-\hat{x}^{(1)}(k-1), k=2,3, \ldots, n
$$

\subsection{Parameter Optimization of Unbiased GM $(1,1)$ Power Model}

We can use the formula (4) to forecast demand if the power exponent $\gamma$ is already known, but $\gamma$ is unknown usually. The value of $\gamma$ is the key factor to affect the prediction accuracy of this model. For this reason, this paper uses the genetic algorithm (GA) method to optimize the unknown parameter $\gamma$ based on minimizing the ARPE.

GA method affects the characteristics string groups without rely on the problem and it searches the possible space of the characteristics strings to find the high-fitness string. The main steps to confirm the best value of $\gamma$ are as follows:

i. Coding

The parameter $\gamma \in[0,2]$ is translated to a binary code string by using the binary coding method. The length of this binary code string is decided by the required precision.

ii. Initialization

Choosing a integer $\mathrm{M}$ act as scale parameter of the population. $\mathrm{M}$ points $\gamma=(\mathrm{i}, 0)(\mathrm{i}=1,2 \ldots, \mathrm{M})$ are selected randomly in $[0,2]$. An original population is composed by these points. Then $\mathrm{P}(0)=\{\gamma=(1,0), \gamma=(2,0), \ldots, \gamma=(\mathrm{K}, 0)\}$.

iii. Determine the fitness function

Calculating the fitness value Fit $[\gamma(\mathrm{i}, \mathrm{k})]$ of each individual $\gamma=(\mathrm{i}, \mathrm{K})$ in the population $\mathrm{P}(\mathrm{k})$, meanwhile $k$ represents algebra (initial $k=0, k \leq K, K$ is an iterative algebra). Fitness function $F(x)$ could be

$$
\text { Fit }[\gamma(i, k)]=\left\{\begin{array}{l}
C_{\max }-f[\gamma(i, k)], C_{\max }-f[\gamma(i, k)]>0, \gamma \in(0,1) \\
0, \text { else }
\end{array}\right.
$$

Meanwhile,

$$
f[\gamma(i, k)]=\frac{1}{n-1} \sum_{n-1}^{n}\left|\frac{\hat{x}^{(0)}(\gamma(i, k))-x^{(0)}(\gamma(i, 0))}{x^{0}(\gamma(i, 0))}\right|
$$

$\hat{x}_{i}^{(0)}(\gamma(i, k))$ act as analog or predicted value of $\gamma(\mathrm{i}, \mathrm{k}), C_{\max }$ is the max APRE in one generation.

iv. Reproduction

The survival probability of each individual is computed in every generation.

$$
p_{i}^{(k)}=\frac{F i t[\gamma(i, k)]}{\sum_{n-1}^{n} \operatorname{Fit}[\gamma(i, k)]}
$$

Furthermore, a random selection stratagem is designed in order to make sure the probability of each individual $\gamma(\mathrm{i}, \mathrm{k})$ will be selected to reproduction is $\mathrm{P}_{\mathrm{i}}^{(\mathrm{k})}$. The individuals, who are generated from propagation, are composited the parent generation $\mathrm{P}(\mathrm{k}+1)$. Crossover is two parent individuals exchange the homologous components under the probability $\mathrm{P}_{\mathrm{c}}$. It also works on the mutation operator when crossover is done. Every individual will be changed on the possibility $\mathrm{P}_{\mathrm{m}}$ in the string.

\section{v. Termination}

Genetic algorithm loop to calculate the fitness value, select Copy, apply crossover and mutation operators. It will terminate until the algorithm to find an acceptable solution, or iterate to preset algebra.

We can follow the general steps of gray prediction to model and forecast when the parameter $\gamma$ is obtained by using Genetic algorithm. This power exponent can reflect the volatility characteristics of the original sequence, and the forecast accuracy could be guaranteed.

\section{Forecasting MRO Spare Parts Inventory}

\subsection{Demand Forecasting for MRO Spare Parts Inventory}

There is the historical demand data of MRO spare parts inventory from one of the biggest steel enterprise in 
china, as Table 1 shows. Here, according to these historical data, we build a traditional GM $(1,1)$ model and a optimized unbiased GM $(1,1)$ power model for one type of important spare parts. The validity and reliability of the optimized model will be verified by forecasting the monthly demand of this kind of spare parts.

Table 1. 2011.01.-2011.10. historical demand quantity of MRO spare parts

\begin{tabular}{ccccccccccc}
\hline Month & 1 & 2 & 3 & 4 & 5 & 6 & 7 & 8 & 9 & 10 \\
\hline Demand quantity & 26 & 20 & 82 & 37 & 78 & 106 & 76 & 71 & 123 & 37 \\
Moving average & & & 42.67 & 46.33 & 65.67 & 73.67 & 86.67 & 84.33 & 90.00 & 77.00 \\
\hline
\end{tabular}

The spare parts of continue casting equipment is very different from normal material. Spare parts services for equipment and stored for maintenance, the lifetime of parts are uncertain, coupled with the order cycle time, cost constraints and other unpredictable factors. So the main features of spare parts are randomness and uncertainty. Compare with the moving average data, the fluctuation of original demand quantity is much bigger, as Figure 1 shows. If we directly use the historical data to build models, the prediction results may not be accurate or the error could be big. In order to keep the high precision and forecast the tendency of spare parts demand, the 10 months demand data should to be processed by using moving average method which is based on the time series, per-series is 3 months.

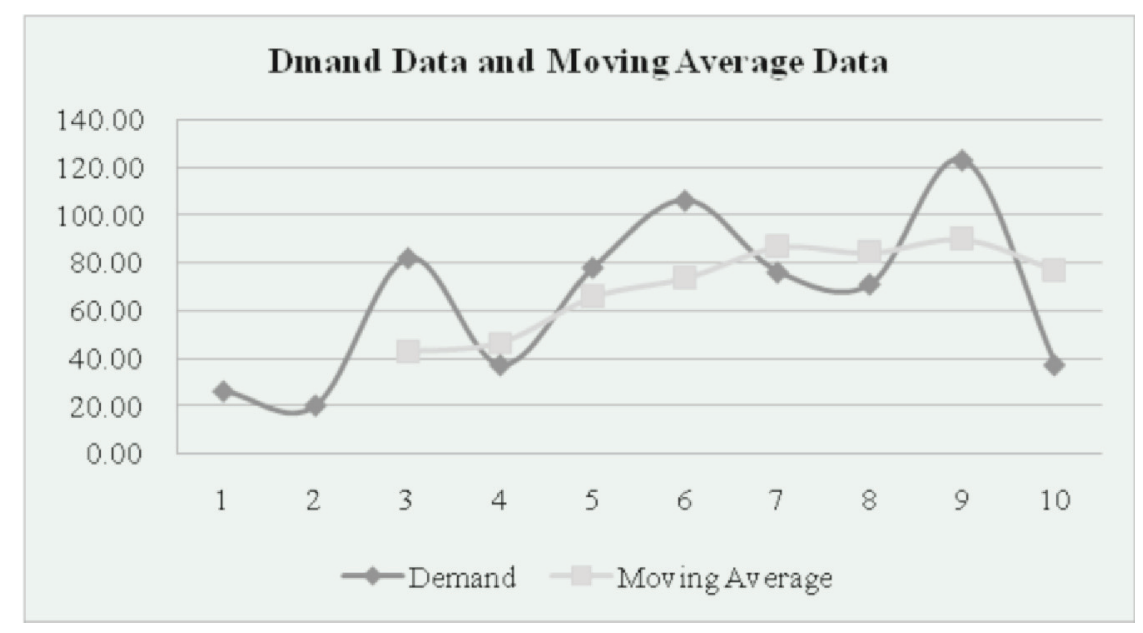

Figure 1. Dmand data and moving average data

Before we calculate the analog and forecasting value, first of all, the power exponent $\gamma$ need to known. The power exponent $\gamma=0$ in the traditional GM $(1,1)$ model, and $\gamma=2$ in the grey Verhulst model, but the real value of $\gamma$ is not that confirm in practice. It is always exist during 0 to 2 . So this paper uses GA method to search the optimum value in the area of $[0,2]$. According to the processed data, one traditional GM $(1,1)$ model and another unbiased GM $(1,1)$ power model are built to forecast spare parts demand. Based on the goal of minimum APRE, when $\gamma=0.9117$, the optimum analog result is found in the unbiased GM $(1,1)$ power model. The analogue data and forecast value are computed, their accuracy is analyzed, and all the results are presented in Table 2.

Table 2. Comparison between two types of model

\begin{tabular}{cccccc}
\hline Month & $\begin{array}{c}\text { Moving } \\
\text { Average } \\
\text { Data }\end{array}$ & \multicolumn{2}{c}{ GM $(\mathbf{1 , 1})$ Model } & \multicolumn{2}{c}{$\begin{array}{c}\text { Unbiased GM }(\mathbf{1}, \mathbf{1}) \text { Power } \\
\text { Model }\end{array}$} \\
\cline { 3 - 6 } & & \multicolumn{2}{c}{$\gamma=0$} & \multicolumn{2}{c}{$\gamma=0.9117$} \\
\cline { 3 - 6 } & & Analog data & RPE (\%) & Analog data & RPE (\%) \\
\hline $\mathbf{3}$ & 42.67 & 42.67 & & 42.67 \\
$\mathbf{4}$ & 46.33 & 61.08 & 31.82 & 45.96 & 0.81 \\
$\mathbf{5}$ & 65.67 & 65.20 & 0.71 & 64.31 & 2.07
\end{tabular}




\begin{tabular}{cccccc}
$\mathbf{6}$ & 73.67 & 69.61 & 5.51 & 78.18 & 6.13 \\
$\mathbf{7}$ & 86.67 & 74.31 & 14.25 & 85.79 & 1.01 \\
$\mathbf{8}$ & 84.33 & 79.34 & 5.92 & 87.24 & 3.44 \\
$\mathbf{9}$ & 90.00 & 84.70 & 5.89 & 83.77 & 6.93 \\
$\mathbf{1 0}$ & 77.00 & 90.42 & 17.43 & 77.00 & 0.00 \\
$\mathbf{1 1}$ & & 96.53 & & 68.45 & \\
$\mathbf{1 2}$ & & 103.06 & & 59.29 & \\
\hline ARPE (\%) & & 11.65 & & 2.91 \\
\hline
\end{tabular}

The differences between the moving average values of demand and analog values are demonstrated clearly by Figue 2.

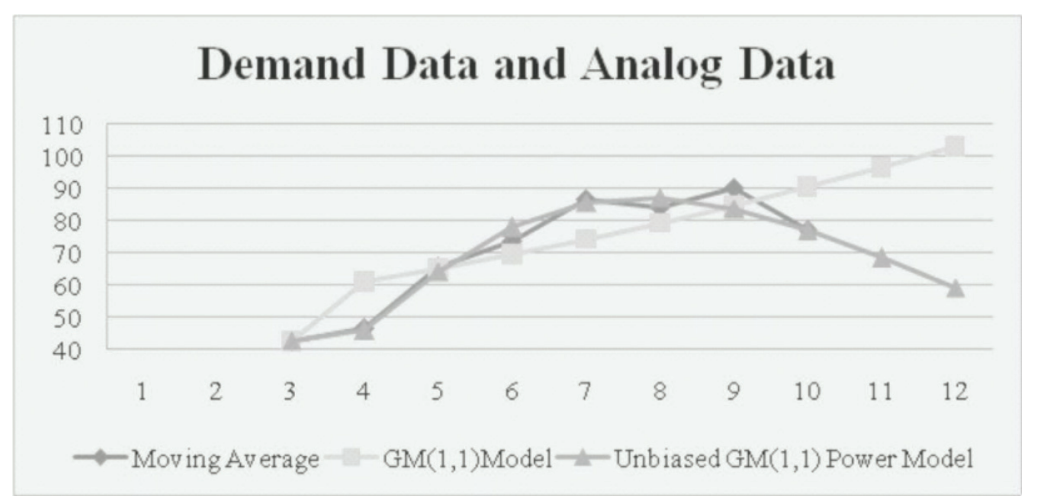

Figue 2. Comparison of demand data and analog data

In the analog values, the data of November and December are the forecast values of these important spare parts. Accurately, the real forecast value should be calculated inversely by moving average method.

\subsection{Result}

In the traditional GM $(1,1)$ model, the analog results of these types of important spare parts, the maximum ARPE is $31.82 \%$, the mean APRE is $11.65 \%$, and the general precision is $88.35 \%$. However, in the optimized unbiased GM $(1,1)$ power model, the maximum APRE of demand is $6.93 \%$, the mean APRE is $2.91 \%$, the general precision is $97.09 \%$ (As shows in Table 2). Compare with these results, the precision of the unbiased GM $(1,1)$ power model is higher than the traditional model. As Figure 2 illustrates, the Curve fitting degree of the unbiased GM $(1,1)$ power model is very good, and it is better than the traditional one's.

Based on the above result, it is shows that the optimized model is effecting to forecast the demand of MRO spare parts, the request of accuracy for demand forecasting of the MRO spare parts inventory for continuous casting equipment in a steel enterprise can be met, and the prediction also can be more stable and trustful.

\section{Conclusions}

This paper research the problem of forecasting which is related to the demand of MRO spare parts for continuous casting equipment of a steel enterprise. Grey theory and GA method were used, and an optimized unbiased GM $(1,1)$ power model was built to forecast. Through the practical example, the forecasting and analog values were calculated and analyzed, and the results indicated that the optimal model can predict the uncertain system and the accuracy also can be guaranteed. Thus, the problem of forecasting accuracy for MRO spare parts can be solved by the optimized unbiased GM $(1,1)$ power model in the steel enterprise, and this method of prediction is practical, valuable and reliable.

\section{References}

Amin-Naseri, M. R., \& Tabar, B. R. (2008). Neural network approach to lumpy demand forecasting for spare parts in process industries. Computer and Communication Engineering, 1378-1382. http://dx.doi.org/ 10.1109/ICCCE.2008.4580831

Chiou, H. K., Tzeng, G., Cheng, C., \& Liu, G. S, (2004). Grey prediction model for forecasting the planning material of equipment spare parts in Navy of Taiwan. Automation Congress, 17(1), 315-320. 
http://dx.doi.org/10.1109/WAC.2004.185428

Deng, J. L. (2002). Grey Theory. Wuhan: Press of Huazhong University of Science \&Technology, Chapter two.

Garg, A., \& Deshmukh, S. G. (2006). Maintenance management: Literature review and directions. Journal of Quality in Maintenance Engineering, 12(3), 205-238. http://dx.doi.org/10.1108/13552510610685075

John, E. B., \& Aris, A. S. (2010). Spare parts management: a review of forecasting research and extensions. Journal of Management Mathematics, 21(3), 227-237. http://dx.doi.org/10.1093/imaman/dpp016

Kostas-Platon, A., Ioulia, M., Rommert, D., \& George, T. (2004). Inventory control of spare parts using a Bayesian approach: A case study. European Journal of Operational Research, 154(1), 730-739. http://dx.doi.org/10.1016/S0377-2217(02)00837-8

Li, D., Yeh, C., \& Chang, C. J. (2009). An improved grey-based approach for early manufacturing data $\begin{array}{lllll}\text { forecasting. Computers } \& \text { Industrial Engineering, } & \text { 57(1), }\end{array}$ http://dx.doi.org/10.1016/j.cie.2009.05.005

Shi, B., Liu, S. F., Dang, Y. G., \& Wang, Z. X. (2011). Recursive Solution to Unbiased Grey Model and Its Optimization. Systems Engineering-Theory \& Practice, 31(8), 1532-1538.

Wang, W. B., \& Aris, A. S. (2011). Spare parts demand: Linking forecasting to equipment maintenance. Transportation Research Part E, 47(1),1194-1209. http://dx.doi.org/10.1016/j.tre.2011.04.008

Wang, Y. F. (2002). Predicting stock price using fuzzy grey prediction system. Expert Systems with Applications, 22(1), 33-39. http://dx.doi.org/10.1016/S0957-4174(01)00047-1

Wang, Z. X., Dang, Y. G., \& Lian, Z. W. (2011). Unbiased GM (1, 1) Power Model and Its Application. Chinese Journal of Management Science, 19(4), 144-151. http://dx.doi.org/CNKI:SUN:ZGGK.0.2011-04-020

Yang, K. L., \& Niu, X. Z. (2009). Research on the Spare Parts Inventory. Industrial Engineering and Engineering Management, 1018-1021. http://dx.doi.org/10.1109/ICIEEM.2009.5344253

Zhang, J. T., \& Lv, B. Y. (2009). Forecasting Intermittent Demand Based on Grey Theory. Intelligent Computation Technology and Automation, 49-52. http://dx.doi.org/10.1109/ICICTA.2009.249 